\title{
Vitrimer Transition Temperature Identification: Coupling Various Thermomechanical Methodologies
}

Amber M. Hubbard ${ }^{1,2,3^{*}}$, Yixin Ren ${ }^{1,2}$, Dominik Konkolewicz ${ }^{4}$, Alireza Sarvestani ${ }^{5}$, Catalin R. Picu ${ }^{6}$, Gary S. Kedziora ${ }^{1}$, Ajit Roy ${ }^{1}$, Vikas Varshney ${ }^{1}$, Dhriti Nepal ${ }^{1 *}$

${ }^{1}$ Materials and Manufacturing Directorate, Air Force Research Laboratory, Wright Patterson Air Force Base, Ohio, United States

${ }^{2}$ ARCTOS, Beavercreek, OH 45432, United States

${ }^{3}$ National Research Council Research Associate, Air Force Research Laboratory, Wright Patterson Air Force Base, Ohio, United States

${ }^{4}$ Department of Chemistry and Biochemistry, Miami University, Oxford, $\mathrm{OH} 45056$, United States

${ }^{5}$ Department of Mechanical Engineering, Mercer University, Macon, GA 31207, United States

${ }^{6}$ Department of Mechanical, Aerospace, and Nuclear Engineering, Rensselaer Polytechnic Institute, Troy, NY 12180, United States

\section{SUPPLEMENTARY INFORMATION}

*Corresponding Authors (amber.hubbard.1.ctr@us.af.mil \& dhriti.nepal.1@us.af.mil) 
a

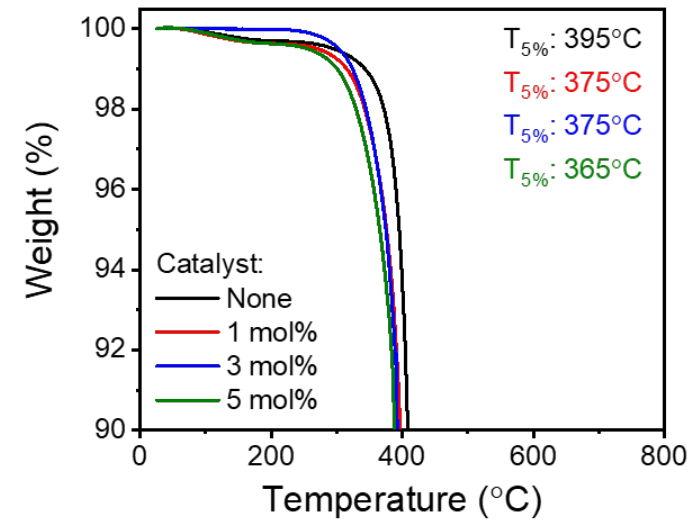

C

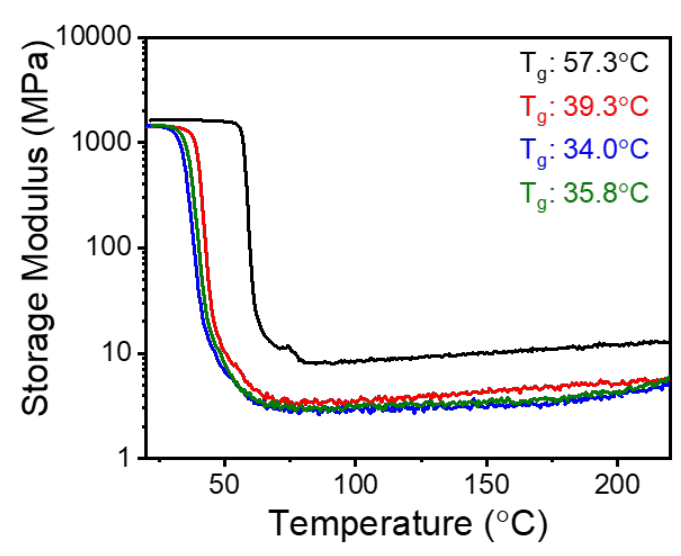

b

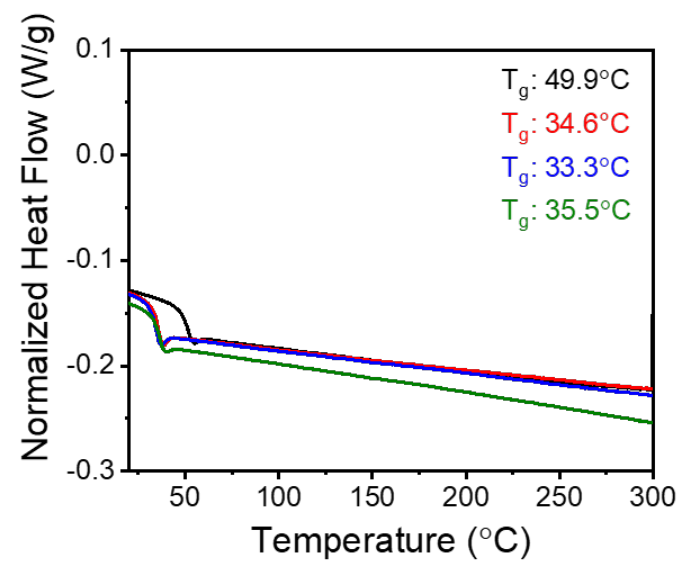

d

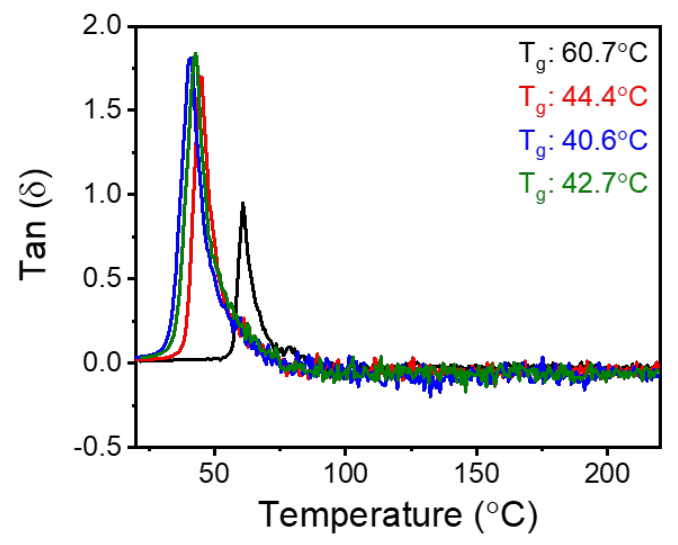

Figure S1. (a) Thermogravimetric analysis (TGA) indicates the temperature at which the vitrimer materials degrade by $5 \mathrm{wt} . \%\left(T_{5 \%}\right) . T_{5 \%}$ is shown here as a function of catalyst concentration. This graph only shows a 10 wt.\% loss for visualization purposes; however, each curve demonstrates a monotonic decrease in weight with a final value $<10 \mathrm{wt} . \%$. A noticeable increase in $T_{5 \%}$ is evident with decreasing catalyst concentration. (b) Differential scanning calorimetry (DSC) shows the glass transition temperature $\left(T_{g}\right)$. While there is a distinct increase in $T_{g}$ in the absence of catalyst, there appears to be a negligible increase in $T_{g}$ with decreasing catalyst concentration. (c-d) Dynamic mechanical analysis (DMA) demonstrates a similar trend in $T_{g}$ as evidenced via DSC. The $T_{g}$ is recorded based on both the (c) storage modulus and (d) $\tan (\delta)$. We note that there is no significant change in the heat flow, storage modulus, or $\tan (\delta)$ once the material reaches the rubbery plateau, indicating that DSC and DMA are not viable methods to identify the $T_{v}$. 

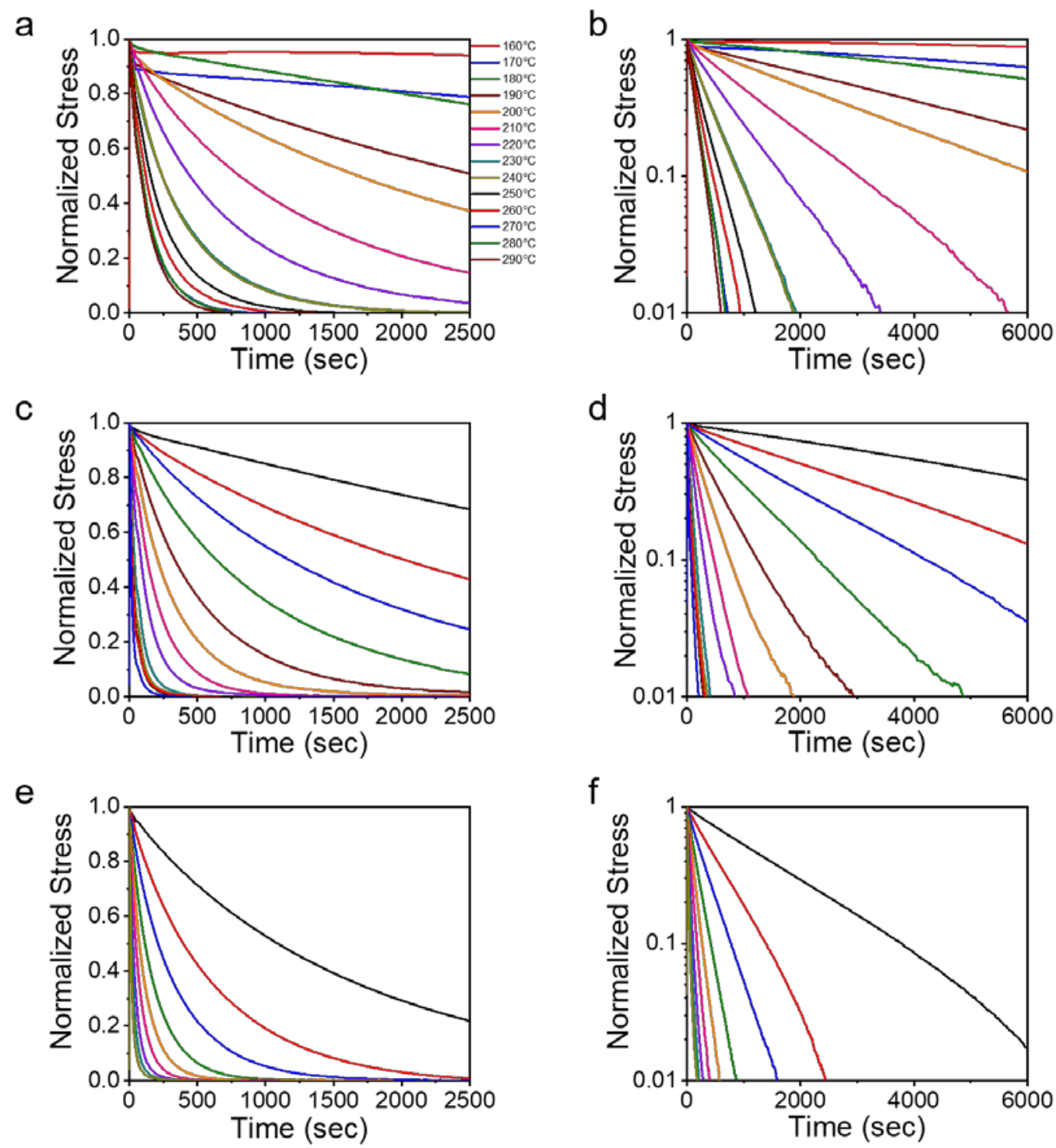

Figure S2. Stress relaxation results are shown for vitrimers with a 1 mol\% (a), 3 mol\% (c), and $5 \mathrm{~mol} \%$ (e) catalyst concentration. In all cases increasing temperature results in faster stress relaxation. The same results are plotted on a logarithmic scale for samples with a $1 \mathrm{~mol} \%$ (b) and $3 \mathrm{~mol} \%$ (d), and $5 \mathrm{~mol} \%$ (f) catalyst concentration. The slight kink in data seen at time $\sim 50$ seconds corresponds to a machine limitation where the time step between recorded data points increases automatically. 
a

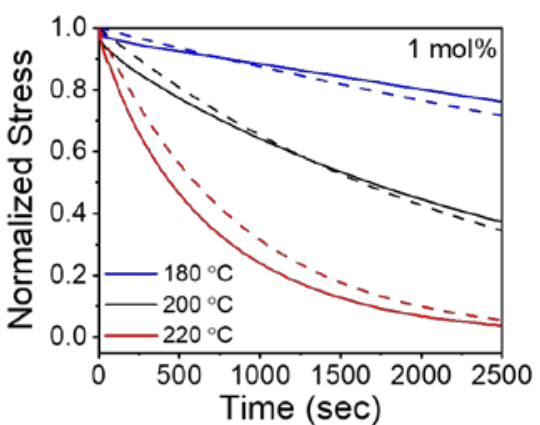

b

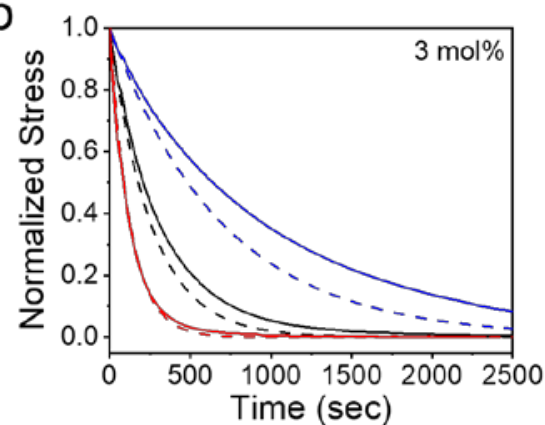

C

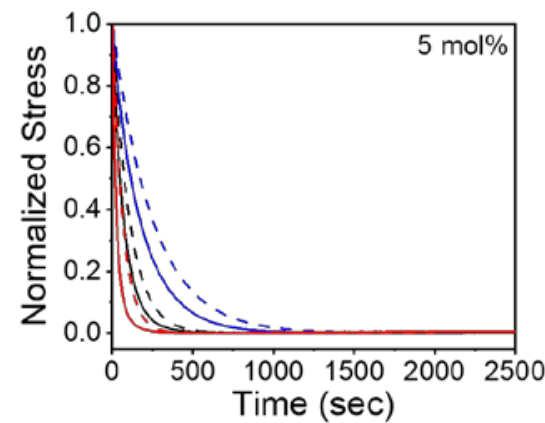

Figure S3. Stress relaxation results for the experimental (solid lines) and theoretical (dashed lines) tests are compared with reasonable agreement; theoretical values are calculated from an exponential function where relaxation times are gathered via Equation 9. Results are shown for samples with catalyst concentrations of (a) 1 mol\%, (b) 3 mol\%, and (c) $5 \mathrm{~mol} \%$ at various temperatures.

\section{Literature Definition of $T_{v, a p p}-$ Derivation}

According to literature, the $T_{v, a p p}$ is defined as the temperature at which the material viscosity reaches $10^{12} \mathrm{~Pa} \mathrm{sec}^{1}$. Using Maxwell's relation for viscoelastic materials, it can be said that

$$
\eta=G \tau
$$

where $\eta$ is viscosity, $G$ is the shear modulus, and $\tau$ is the stress relaxation constant. To relate this equation to measured material properties for this homogeneous isotropic material we can say

$$
G=\frac{E^{\prime}}{2(1+v)}
$$

where $E^{\prime}$ is the storage modulus and $v$ is the Poisson's ratio. Using DMA (cf. Figure S1c), $E^{\prime}$ is recorded as $2.17 \mathrm{MPa}$ while $v$ is assumed to be 0.5 as is common for polymer materials in the rubbery regime. Combining Equations S1 \& S2 to solve for $\tau$ yields Equation S3,

$$
\tau=\frac{2(1+v) \eta}{E^{\prime}}
$$

where calculating $\tau$, for $\eta=10^{12} \mathrm{~Pa} \mathrm{sec}$, results in $\sim 10^{6} \mathrm{sec}^{2,3}$. Intersecting the linear fit of the Arrhenius plot with this calculated $\tau$ yields a $T_{v, \text { app }}$ of $81.0^{\circ} \mathrm{C}$, as seen in Figure $1 \mathrm{e}$. 


\section{Kinetic Model - Curve Fitting}

The curve fitting of Equation $\mathbf{9}$ to the stress relaxation data within Figure $\mathbf{2}$ was done using a curve_fit function via the SciPy library in Python. The unified equation originally had an undesirable bias in the calculated parameters, due to the fact that each curve contained a different number of data points $(14,13$, and 9 data points for 1,3 , and 5 mol\% catalyst concentrations, respectively). In order to minimize this bias, a random_choice function was applied so that 9 random points would be chosen from each data set representing the various catalyst concentrations; this number of data points was chosen to reduce bias while maximizing the use of the empirical data. As the calculated parameter fits from this curve fitting would vary with each calculation based on the randomized data selection, the curve fitting was performed $\sim 2,000$ times. The average and standard deviation values for each fit parameter are reported in the main text.

\section{Kinetic Model - Derivation for the Diffusion-limited Regime}

Equation 8 defines the rate constant for the diffusion-limited regime of the Arrhenius plots as seen in Figure 2. Within this regime, a phenomenological model is implemented assuming Rouse-like chain relaxation. For Rouse-like chain mobility there is a known relationship between the characteristic time for diffusion and the molecular weight and temperature so that we can say

$$
\begin{aligned}
\tau_{D} & \propto N^{2} \\
\tau_{D} & \propto \frac{1}{T}
\end{aligned}
$$

where $\tau_{D}$ is the time constant for chain diffusion, $N$ is the molecular weight, and $T$ is the temperature. Combining Equations S4 and S5 yields:

$$
\tau_{D} \propto \frac{N^{2}}{T}
$$

Within this high-temperature regime, the effective molecular weight between the transesterification reaction sites is a function of the catalyst concentration. In other words, increasing catalyst concentration increases the number of transesterification reactions (and reaction sites), resulting in an effectively shortened polymer strand (i.e., an effectively lowered molecular weight). In terms of its dependence on catalyst concentration, this effective length of a Rouse chain takes the functional form of

$$
N \propto \frac{1}{\sqrt[3]{C}}
$$

where $C$ is the catalyst concentration. As the catalyst concentration decreases, $N$ approaches infinity, representing the molecular weight of a fully cross-linked polymer 
network. The inverse cube-root relationship between $N$ and $C$ represents the volume relationship of the catalyst; as catalyst concentration increases by 8 , the effective molecular weight between reaction sites decreases by 2 . This relationship between $N$ and $\mathbf{C}$ is depicted visually in Figure $\mathbf{S 4}$, where each node (i.e., grey dot) represents a reaction site and the lines connecting each reaction site represents the effective molecular weight.
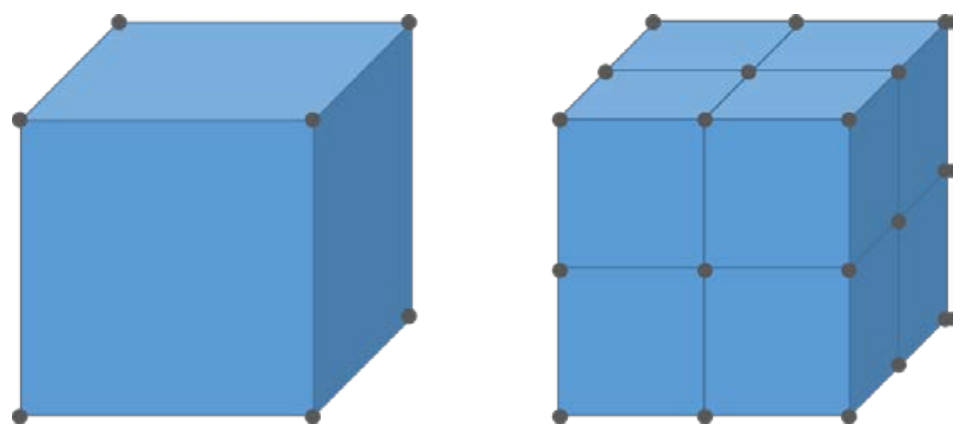

Figure S4. A set of cubes visually depicts the relationship between $N$ and $C$. In this diagram, each grey dot represents a transesterification reaction site.

Combining Equations $\mathbf{S 6}$ and $\mathbf{S 7}$ yields:

$$
\tau_{D} \propto \frac{1}{T C^{2 / 3}}
$$

In addition, combining Equations 6 and 8, yields:

$$
\tau_{D}=\frac{1}{k_{D}}=\frac{1}{A_{D} C^{2} / 3 T}
$$

where $k$ is the rate constant and $A_{D}$ is the proportionality constant for the diffusion-limited regime. Extrapolating from Equation $\mathbf{S 8}$ and $\mathbf{S 9}$, the prefactor $A_{D}$ can be associated with a variety of chain properties (e.g., intrinsic flexibility of the diffusing strands, strength of the inter-chain interactions, etc.), which can modulate the effective relaxation; meanwhile, the catalyst exponent can be taken as $2 / 3$. It should be noted that the catalyst exponent for the diffusion-limited regime (2/3) found in Equations $\mathbf{8}$ and $\mathbf{S 9}$ was also tested as a free parameter when inputted to the multi-objective fitting function. Under such fitting, this exponent was estimated to be $0.68 \pm 0.03$ providing additional confidence to our phenomenological model.

\section{Kinetic Model - Physical Insights}

While increasing catalyst concentration is expected to accelerate stress relaxation, its observed effect is much more pronounced than linear scaling, as evidenced by the exponent $b$ equaling $2.24 \pm 0.02$. For the local network structure to undergo measurable stress relaxation, it is reasonable to assume that multiple transesterification reactions 
must occur simultaneously within a localized region. Increasing the catalyst concentration significantly increases the probability of transesterification reactions taking place within a particular chain, thus increasing the overall likelihood of network relaxation. Therefore, increasing both catalyst concentration and temperature results in a synergistic effect upon stress relaxation.

Figure 2a-c suggests that additional stress relaxation experiments would be required to reach the true diffusion-limited regime. However, as a result of physical limitations, it is impossible to extend the upper temperature of our tests for each catalyst concentration due to material degradation and sample adhesion to the testing apparatus.

Within the investigated temperature range, it should also be noted that the diffusion-limited regime plateau increases with decreasing catalyst concentration (cf. Figure 2d) as seen in both the experimental data and the kinetic model. Here, the stress relaxation is controlled by the dynamic reconfiguration of reactive polymer chains, whose effective chain length depends on the catalyst concentration as stated in the above section. Increasing the catalyst concentration increases the number of reactions taking place within the network per unit volume, thus reducing the characteristic length scale of the diffusing segment (cf. Figure S4). Following a transesterification reaction, this polymer chain becomes "free" to reconfigure/diffuse within the network as a means to reduce applied stress. Increasing the catalyst concentration, and therefore the number of transesterification reactions, effectively shortens the polymer chain undergoing reconfiguration; this effect increases overall chain mobility increasing the rate of stress relaxation, lowering the observed relaxation time. In addition, the scaling of the relaxation time with respect to the catalyst concentration is explored graphically in Figure S5. 


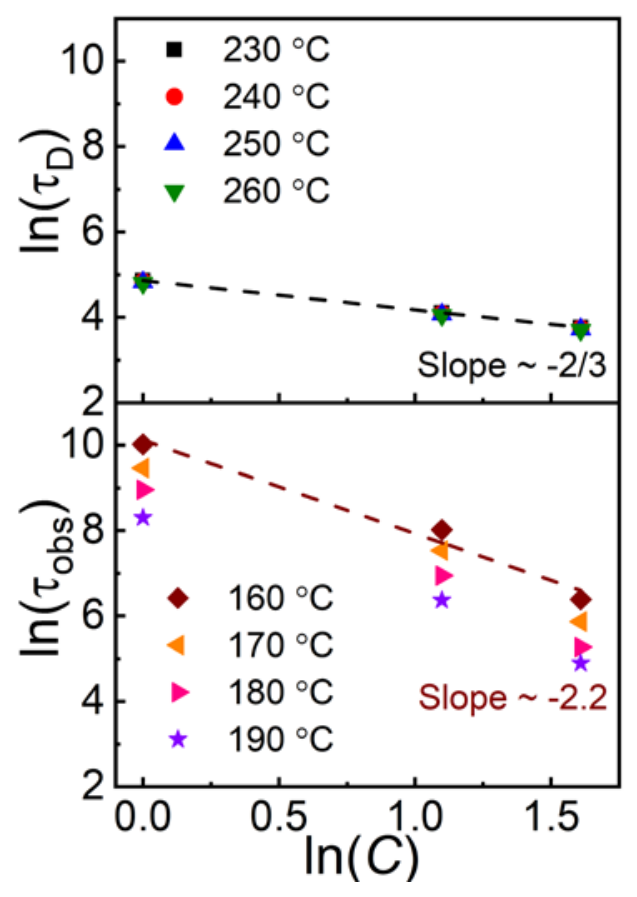

Figure S5. The relationship between the stress relaxation time and the catalyst concentration is presented graphically for both the low- and high-temperature regimes. 

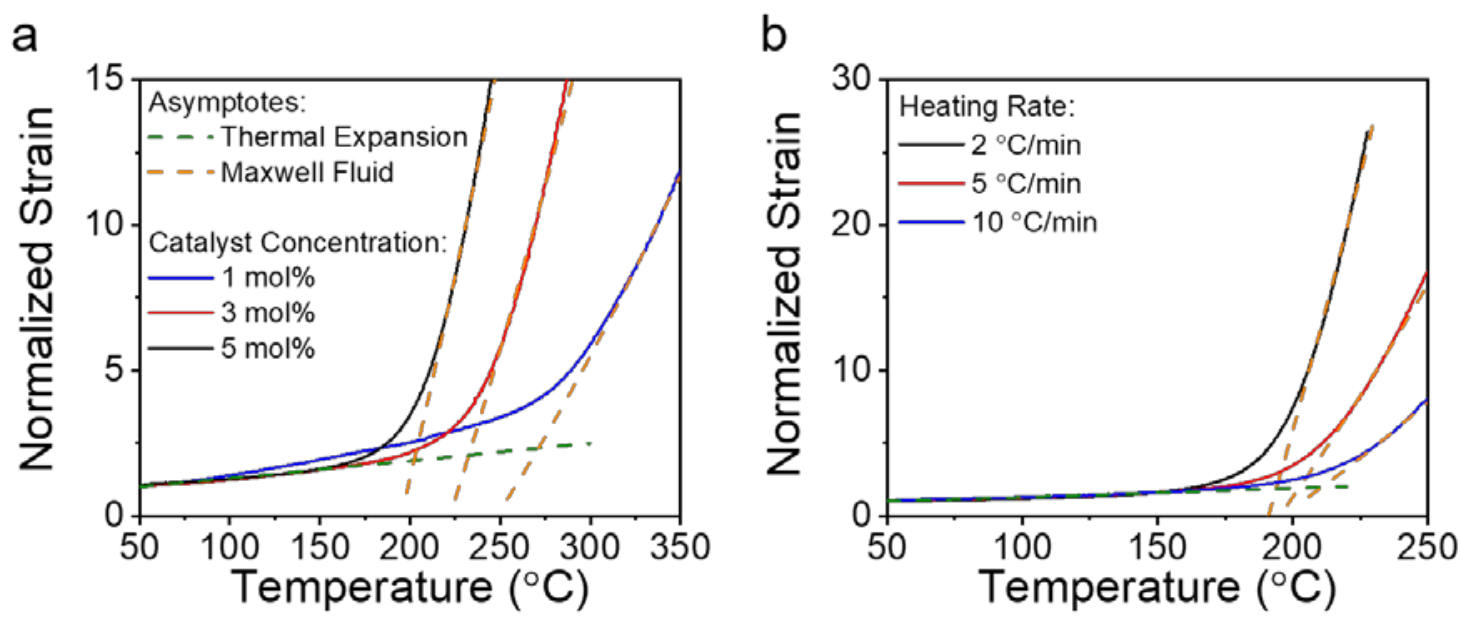

Figure S6. Non-isothermal creep results are shown for neat vitrimer samples with (a) varying catalyst concentrations at a heating rate of $5{ }^{\circ} \mathrm{C} \mathrm{min}-1$ and (b) varying heating rates at a $5 \mathrm{~mol} \%$ catalyst concentration. Herein, the strain is normalized by the equilibrium strain. It is important to note that all non-isothermal creep results exhibit similar slopes in the low-temperature regime; in this regime, the pseudo-horizontal dashed, green line indicates the expected increase in strain as a result of thermal expansion for an epoxy material $\left.\right|^{4,5}$. In the high temperature region, the orange, dashed line is directly proportional to $\beta^{-1} \tau_{D}{ }^{-1}$, where $\beta$ is the heating rate, indicating the vitrimer is behaving as a Maxwell fluid. These asymptotic slopes are consistent regardless of the experimental conditions and we postulate the $T_{v}$ is the intersection of these two asymptotes. 

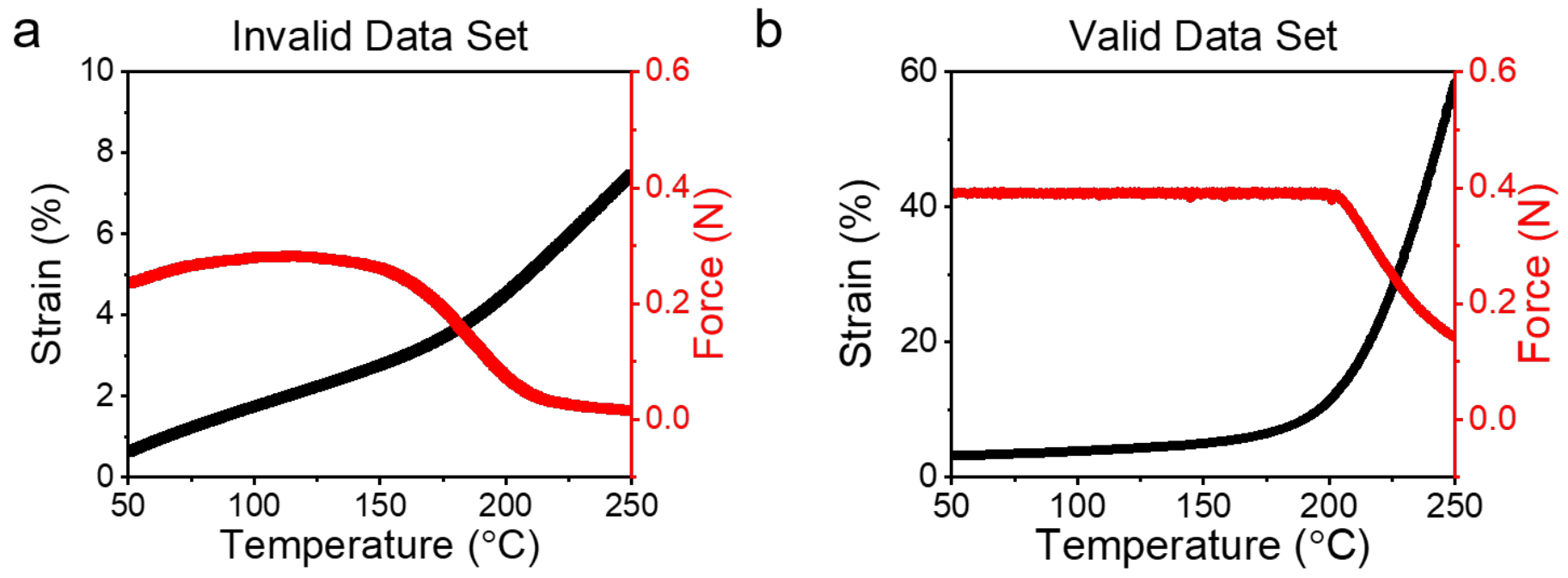

Figure S7. (a) A failed non-isothermal creep experiment results in a force curve which does not remain constant. This experiment was performed on a vitrimer sample with a 5 mol\% catalyst concentration. The temperature ramp rate was $5^{\circ} \mathrm{C} \mathrm{min}-1$ with an applied axial force of $0.5 \mathrm{~N}$. (b) A comparable test was performed resulting in a valid data set; test validity is confirmed by a constant tensile force being maintained on the sample. 
a

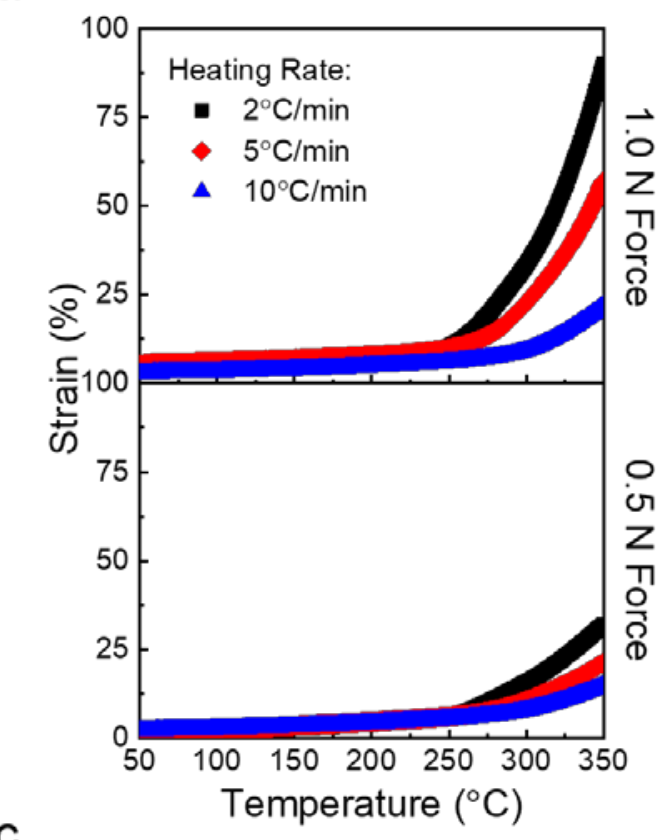

C

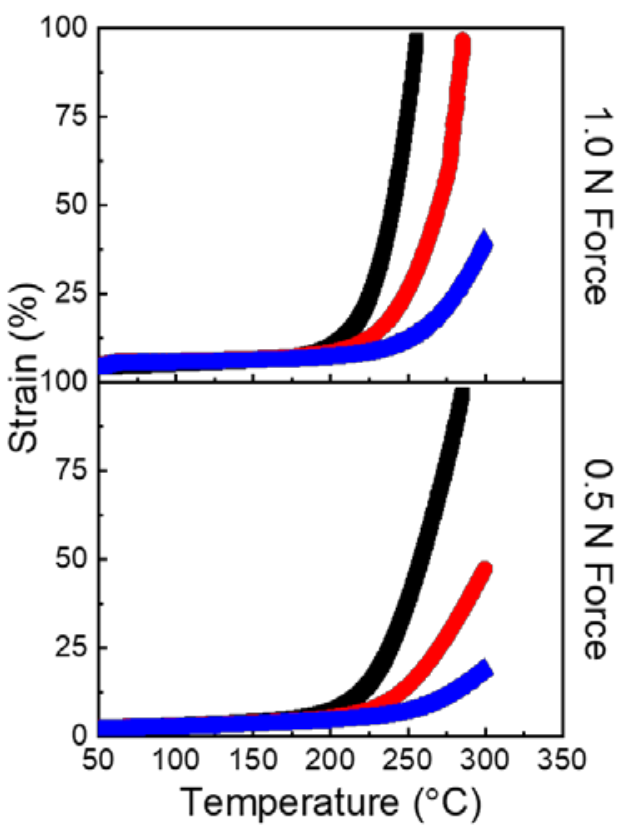

b
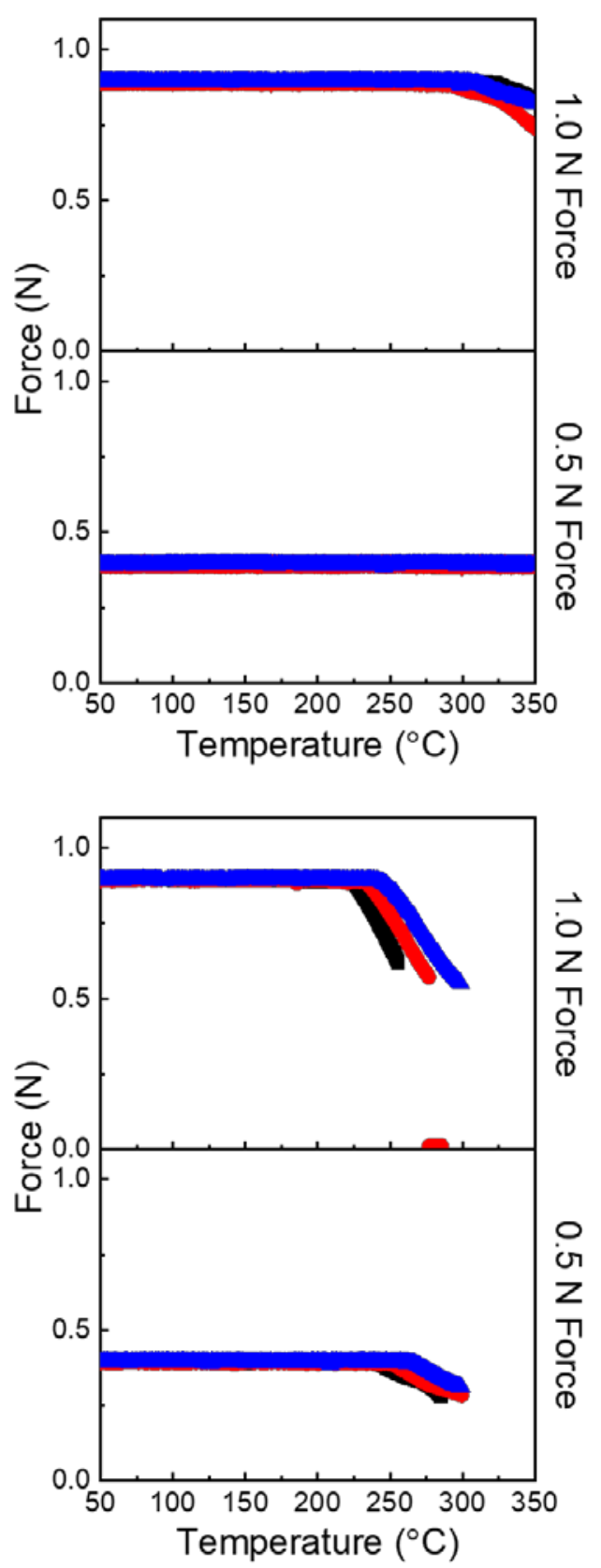

Figure S8. Non-isothermal creep results for vitrimer samples with a 1 mol\% (a \& b) and $3 \mathrm{~mol} \%$ ( $c$ \& d) catalyst concentration demonstrate that increasing heating rate and decreasing applied force increase the measured $T_{v}$. The data for samples with a $1 \mathrm{~mol} \%$ catalyst concentration is slightly noisy and, at sufficiently low applied axial force, there is no decrease in the force-temperature curve over the tested temperature range. We postulate this is due to a sufficiently low number of transesterification reactions occurring at sufficiently low applied forces, resulting in an unmeasurable change. 
a

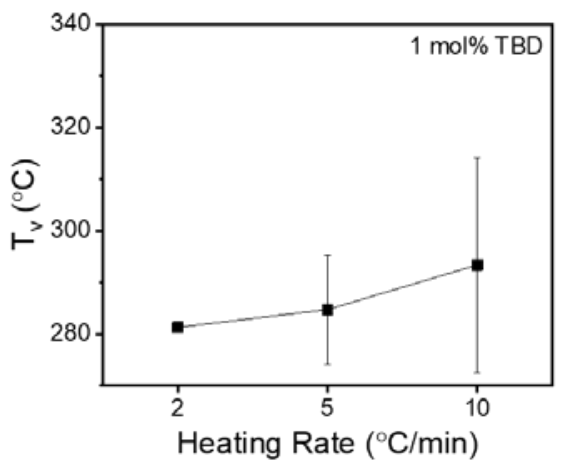

C

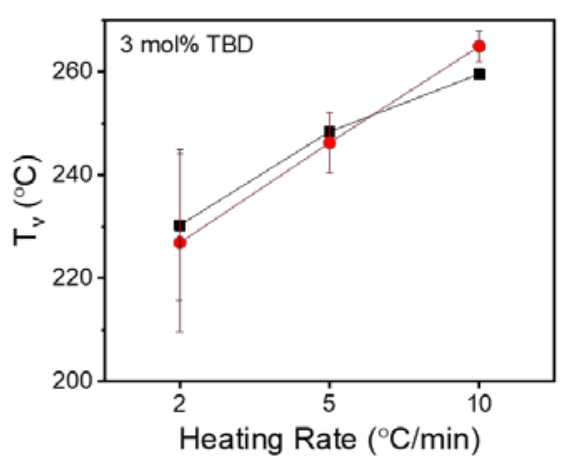

e

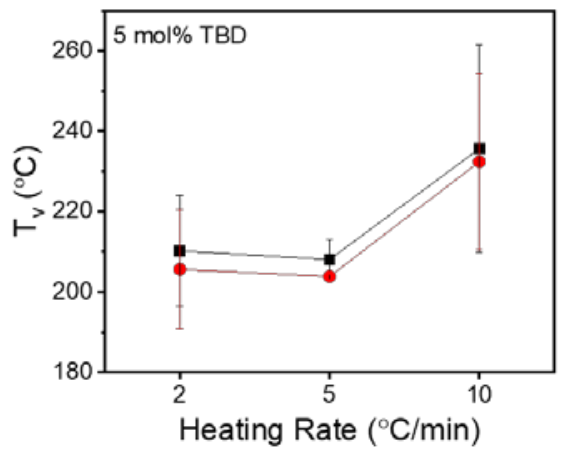

b

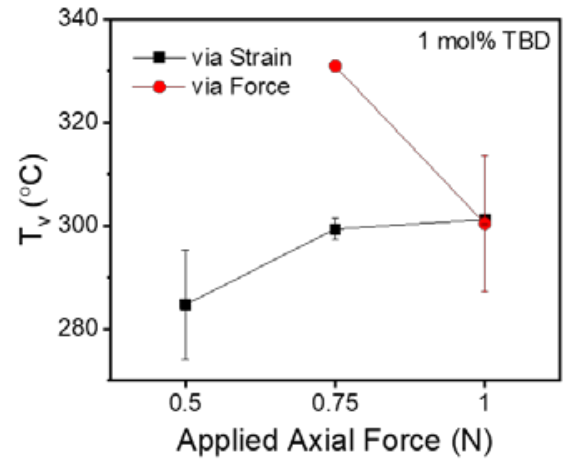

d

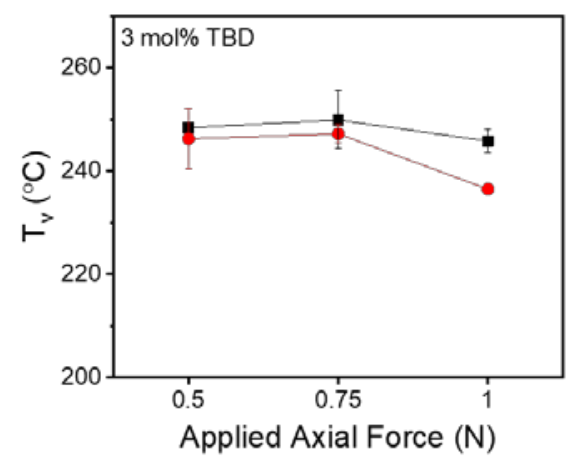

f

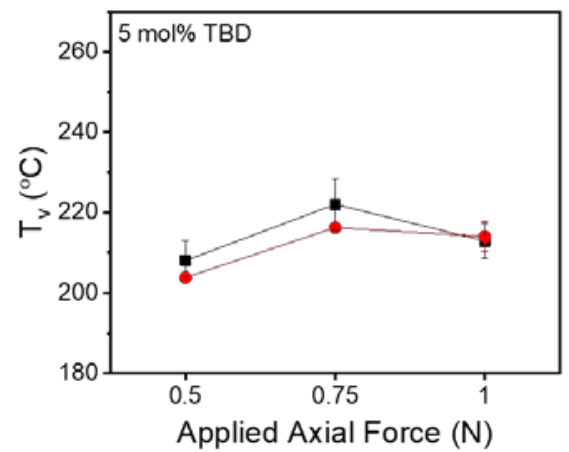

Figure S9. Non-isothermal creep experiments quantitatively demonstrate the impact of heating rate and applied axial force on the $T_{v}$ as measured via strain (black data) and force (red data) curves. Increasing the heating rate and decreasing the applied force increases the recorded $T_{v}$. These trends hold for 1 mol\% ( $a$ \& b), 3 mol\% (c \& d), and 5 mol\% (e \& f) catalyst concentrations. For the plots where the heating rate is varied (a, $c$, $\&$ e), the applied axial force is $0.5 \mathrm{~N}$. For the plots where the applied axial fore is varied (b, d, \& f), the heating rate is $5^{\circ} \mathrm{C} \mathrm{min}-1$. It should be noted that the force curve is not an indicator of $T_{v}$ for some tests where insufficient force and temperature ranges are applied, which results in "missing" data points for samples with a 1 mol\% catalyst concentration (a \& b). 
a

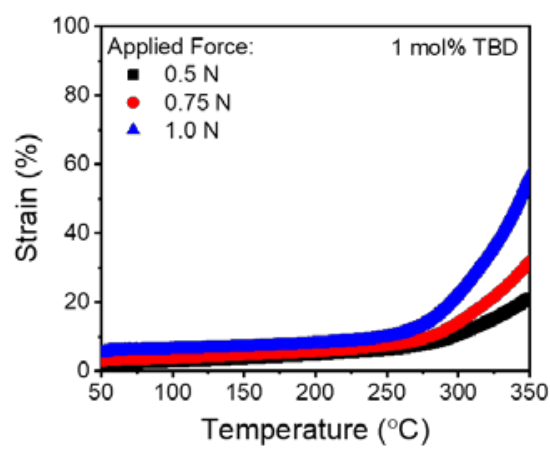

C

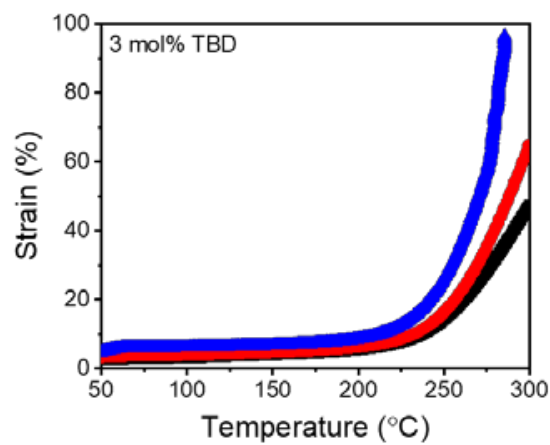

e

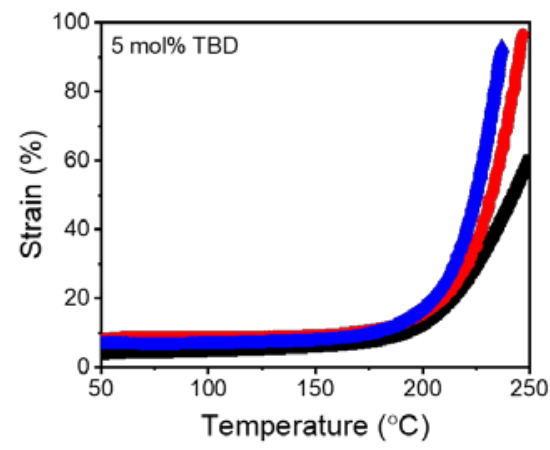

b

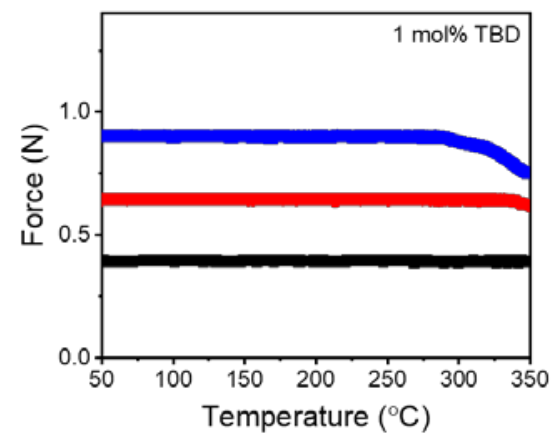

d

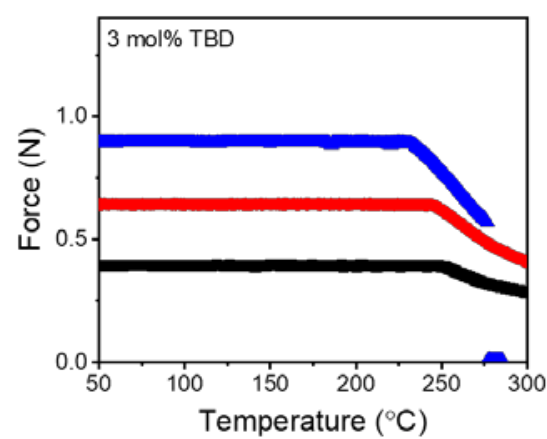

f

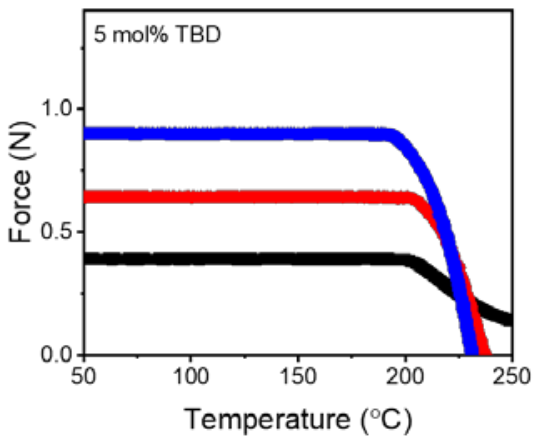

Figure S10. Non-isothermal creep experiments demonstrate qualitatively that increasing applied axial force decreases the measured $T_{v}$. This trend is consistent for vitrimer samples with a 1 mol\% (a \& b), 3 mol\% (c \& d), and 5 mol\% (e \& f) catalyst concentration. 
a

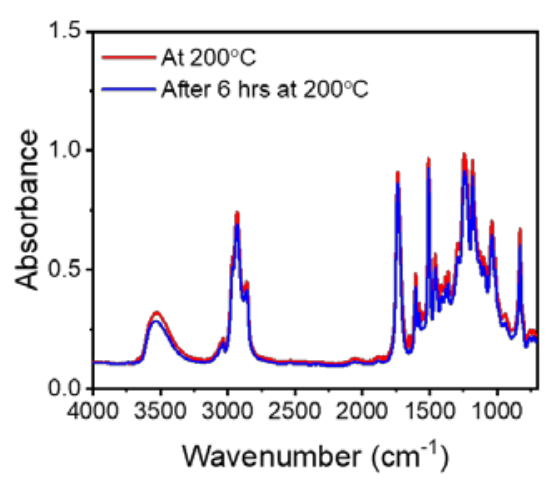

C

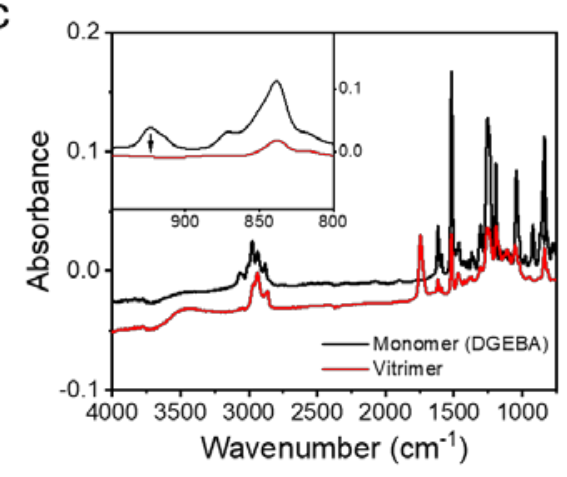

b

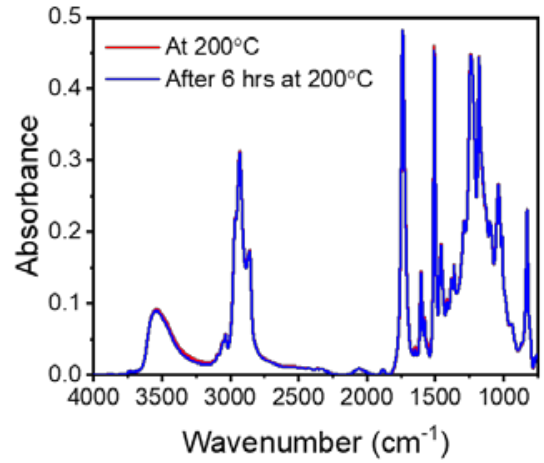

d

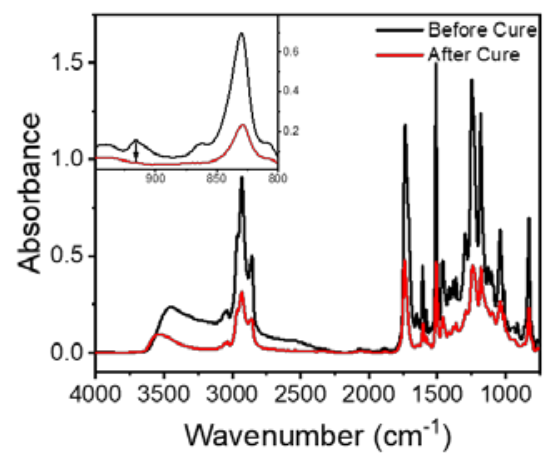

Figure S11. FTIR results are shown for vitrimer systems (a) without and (b) with catalyst present. In-situ FTIR was collected at an elevated temperature of $200{ }^{\circ} \mathrm{C}$ for six hours to track chemical changes in the system. By plotting the initial and final spectra, we confirm a lack of side reactions in the material by negligible changes in the spectra. FTIR results are shown comparing the results of the cured vitrimer against the (c) monomer and (d) uncured reaction mixture. The significant peak decrease around $915 \mathrm{~cm}^{-1}$, as seen in the inset of each graph, confirms epoxide ring deformation indicating monomer consumption ${ }^{6-}$ 8 .

\section{References}

1 Montarnal, D., Capelot, M., Tournilhac, F. \& Leibler, L. Silica-Like Malleable Materials from Permanent Organic Networks. Science 334, 965-968 (2011).

2 Capelot, M., Unterlass, M. M., Tournilhac, F. \& Leibler, L. Catalytic Control of the Vitrimer Glass Transition. ACS Macro Letters 1, 789-792 (2012).

3 Chen, M., Zhou, L., Wu, Y., Zhao, X. \& Zhang, Y. Rapid Stress Relaxation and Moderate Temperature of Malleability Enabled by the Synergy of Disulfide Metathesis and Carboxylate Transesterification in Epoxy Vitrimers. ACS Macro Letters 8, 255-260 (2019).

4 Singer, G., Sinn, G., Lichtenegger, H. C., Veigel, S., Zecchini, M. \& Wan-Wendner, R. Evaluation of in-situ shrinkage and expansion properties of polymer composite 
materials for adhesive anchor systems by a novel approach based on digital image correlation. Polymer Testing 79, 8 (2019).

5 Davis, G. D. in Handbook of Adhesive Technology Ch. 13, (Marcel Dekker, Inc., 2003).

6 Manakhov, A., Fukova, S., Necas, D., Michlicek, M., Ershov, S., Elias, M., Visotin, M., Popov, Z. \& Zajickova, L. Analysis of epoxy functionalized layers synthesized by plasma polymerization of allyl glycidyl ether. Phys Chem Chem Phys 20, 2007020077 (2018).

7 Nikolic, G., Zlatkovic, S., Cakic, M., Cakic, S., Lacnjevac, C. \& Rajic, Z. Fast Fourier transform IR characterization of epoxy GY systems crosslinked with aliphatic and cycloaliphatic EH polyamine adducts. Sensors (Basel) 10, 684-696 (2010).

8 Huang, J. \& Nie, X. A simple and novel method to design flexible and transparent epoxy resin with tunable mechanical properties. Polymer International 65, 835-840 (2016). 